\title{
Quasi-periodic Solutions of a Spiral Type for Photogravitational Restrict- ed Three-Body Problem
}

\author{
Sergey V. Ershkov*
}

Institute for Time Nature Explorations, M.V. Lomonosov's Moscow State University, Leninskie gory, 1-12, Moscow 119991, Russia

\begin{abstract}
A new type of exact solutions for photogravitational restricted three-body problem (a case of spiral motion) is presented here.

A key point is that we obtain the appropriate specific case of spiral motions from the Jacobian-type integral of motion for photogravitational restricted three-body problem (when orbit of small 3-rd body is assumed to be like a spiral).

Besides, we should especially note that there is a proper restriction to the type of spiral orbital motion of small 3-rd body, which could be possible for choosing as the exact solution of equations for photogravitational restricted three-body problem.

The main result, which should be outlined, is that in a case of quasi-planar orbital motion (of the small 3-rd body) the asymptotic expression for component $Z$ of motion is proved to be given by the proper elliptical integral.
\end{abstract}

Keywords: Photogravitational restricted three-body problem, spiral motion, Jacobian-type integral of motion.

\section{INTRODUCTION}

In this contribution, we present a new type of exact solutions for photogravitational restricted three-body problem [1-3], which is the case of spiral motions.

According to the Bruns theorem [4], there is no other invariants except well-known 10 integrals for three-body problem (including integral of energy, momentum, etc). But in the case of restricted three-body problem, there is no other invariants except only one, Jacobian-type integral of motion $[5,6]$.

The main idea is to obtain the appropriate specific case of spiral motion for photogravitational restricted three-body problem from the Jacobian-type integral of motion (when orbit of small 3-rd body is assumed to be like a spiral); besides, such a case of spiral motion should be adopted by the structure of the Jacobian-type integral of motion.

In addition, we should emphasize the appropriate astrophysical application of the constructed (exact) solutions of a spiral motion: for example, we could consider the SunJupiter system as primaries and assume that only the larger primary (Sun) radiates. Besides, we could consider small objects such as meteoroids or small asteroids (about $10 \mathrm{~cm}$ to $10 \mathrm{~km}$ in diameter) as the small 3-rd body for such a case.

*Address correspondence to this author at the Institute for Time Nature Explorations, M.V. Lomonosov's Moscow State University, Leninskie gory, 1-12, Moscow 119991, Russia; Tel: +7-916-338-66-97;

E-mail: sergej-ershkov@yandex.ru

\section{EQUATIONS OF MOTION}

Let us consider the system of ODE for photogravitational restricted three-body problem, at given initial conditions [2].

We consider three bodies of masses $m 1, m_{2}$ and $m$ such that $m_{1}>m_{2}$ and $m$ is an infinitesimal mass. The two primaries $m 1$ and $m 2$ are sources of radiation; $q 1$ and $q_{2}$ are factors of the radiation effects of the two primaries respectively, $\left\{q_{1} 1, q_{2}\right\} \in(-\infty, 1]$.

We assume that $m 2$ is an oblate spheroid. The effect of oblateness $[7,8]$ is denoted by the factor $A 2$.

Let $r i(i=1,2)$ be the distances between the centre of mass of the bodies $m r$ and $m 2$ and the centre of mass of body $m$. The unit of mass is chosen so that the sum of the masses of finite bodies becomes equal to 1 .

We suppose that $m_{1}=1-\mu$ and $m_{2}=\mu$, where $\mu$ is the ratio of the mass of the smaller primary to the total mass of the primaries and $0 \leq \mu \leq 0,5$. The unit of distance is taken as the distance between the primaries. The unit of time is chosen so that the gravitational constant is equal to 1 .

The three dimensional restricted three-body problem, with an oblate primary $m 2$ and both primaries radiating, could be presented in barycentric rotating co-ordinate system by the equations of motion below [7, 8]:

$$
\begin{aligned}
\ddot{x}-2 n \dot{y} & =\frac{\partial \Omega}{\partial x}, \\
\ddot{y}+2 n \dot{x} & =\frac{\partial \Omega}{\partial y}, \\
\ddot{z} \quad & =\frac{\partial \Omega}{\partial z},
\end{aligned}
$$




$$
\begin{aligned}
& \Omega=\frac{n^{2}}{2}\left(x^{2}+y^{2}\right)+\frac{q_{1}(1-\mu)}{r_{1}}+ \\
& \frac{q_{2} \mu}{r_{2}}\left[1+\frac{A_{2}}{2 r_{2}^{2}} \cdot\left(1-\frac{3 z^{2}}{r_{2}^{2}}\right)\right]
\end{aligned}
$$

where

$$
n^{2}=1+\frac{3}{2} A_{2}
$$

is the angular velocity of the rotating coordinate system and A 2 - is the oblateness coefficient. Here

$$
A_{2}=\frac{A E^{2}-A P^{2}}{5 R^{2}}
$$

where $A E$ is the equatorial radius, $A P$ is the polar radius and $R$ is the distance between primaries. Besides, we should note that

$$
\begin{aligned}
& r_{1}^{2}=(x+\mu)^{2}+y^{2}+z^{2}, \\
& r_{2}^{2}=(x-1+\mu)^{2}+y^{2}+z^{2},
\end{aligned}
$$

are the distances of infinitesimal mass from the primaries.

We neglect the relativistic Poynting-Robertson effect [910] which may be treated as a perturbation for cosmic dust or for small particles (less than $1 \mathrm{~cm}$ in diameter), we neglect the Yarkovsky effect of non-gravitational nature [11-13], as well as we neglect the effect of variable masses of 3-bodies $[14,15]$.

The possible ways of simplifying of equations (2.1):

if we assume that the effect of oblateness is zero, $A_{2}=0$ ( $\Rightarrow n=1$ ), it means $m 2$ is non-oblate spheroid (we will consider only such a case below);

if we assume $q_{1}=q_{2}=1$, it means a case of restricted three-body problem.

\section{EXACT SOLUTION (A CASE OF SPIRAL MOTION)}

Regarding the orbit of small 3-rd body, let us assume such an orbit to be presented like a spiral (Fig. 1).

Besides, let us remind that we could obtain from the equations of system (2.1) a Jacobian-type integral of motion $[5,6]$ :

$$
(\dot{x})^{2}+(\dot{y})^{2}+(\dot{z})^{2}=2 \Omega(x, y, z)+C
$$

where $C$ is so-called Jacobian constant.

As per assumption above, it means that components of solution $\left\{x_{i}\right\}=\{x(t), y(t), z(t)\}(i=1,2,3)$ should be presented as below:

$$
x=\xi(t) \cdot \cos (w \cdot t), \quad y=\xi(t) \cdot \sin (w \cdot t), \quad z=z(t),(*)
$$

where the angular velocity is chosen $w=1 ; \xi(t)$ - is a spiral factor. For example:

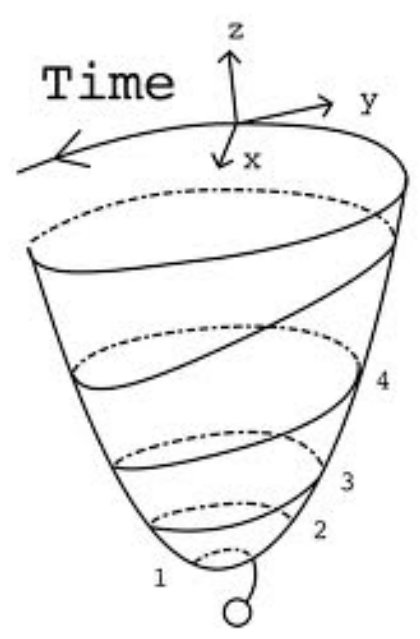

Fig. (1). Type of spiral motion.

1) If $\xi(t)=a \cdot t+c, z(t)=b \cdot t$ - we should obtain the spiral of screw line type,

2) If $\xi(t)=a \cdot \exp (b \cdot t), z(t)=c \cdot t$ - we should obtain the $3-D$ logarithmic spiral,

here $\{a, b, c\}$ are supposed to be the arbitrary positive real constants.

Thus if we substitute the representation $(*)$ for the components of solution $\{x i\}=\{x(t), y(t), z(t)\}$ into the Equation (3.1), the following equation is obtained

$$
\begin{aligned}
& (\dot{\xi}(t) \cdot \cos t-\xi(t) \cdot \sin t)^{2}+(\dot{\xi}(t) \cdot \sin t \\
& +\xi(t) \cdot \cos t)^{2}+(\dot{z})^{2}=2 \Omega(x, y, z)+C \\
& \Rightarrow \quad \dot{\xi}^{2}(t)+\xi^{2}(t)+(\dot{z})^{2}=2 \Omega(x, y, z)+C
\end{aligned}
$$

where the expression for $\Omega(t)$ in (2.2) should be simplified in the case of non-oblateness $A 2=0(n=1)$ :

$$
\begin{aligned}
& \Omega(t)=\frac{\xi^{2}(t)}{2}+\frac{q_{1}(1-\mu)}{r_{1}}+\frac{q_{2} \mu}{r_{2}}, \\
& r_{1}{ }^{2}=(\xi(t) \cdot \cos t+\mu)^{2}+(\xi(t) \cdot \sin t)^{2}+z(t)^{2}, \\
& r_{2}{ }^{2}=(\xi(t) \cdot \cos t-1+\mu)^{2}+(\xi(t) \cdot \sin t)^{2}+z(t)^{2} .
\end{aligned}
$$

So, taking into consideration the expression (3.3) for $\Omega$ $(t)$, we obtain from (3.2)

$$
\dot{\xi}^{2}(t)+(\dot{z})^{2}=\frac{2 q_{1}(1-\mu)}{r_{1}}+\frac{2 q_{2} \mu}{r_{2}}+C
$$

$r_{1}^{2}=(\xi(t) \cdot \cos t+\mu)^{2}+(\xi(t) \cdot \sin t)^{2}+z(t)^{2}$,

$$
r_{2}^{2}=(\xi(t) \cdot \cos t-1+\mu)^{2}+(\xi(t) \cdot \sin t)^{2}+z(t)^{2} \text {. }
$$

Besides, we should note from (3.4) that the proper restriction below should be valid: 


$$
\frac{2 q_{1}(1-\mu)}{r_{1}}+\frac{2 q_{2} \mu}{r_{2}}+C \geq 0
$$

here $\left\{q_{1}, q_{2}\right\} \in(-\infty, 1]$. There are two possibilities to solve the equation (3.4):

1) First, we assume $\mathrm{z}(t)$ to be given as a proper function of parameter $t$, then we should obtain a solution of ODE of the 1-st kind for $\xi(t)$;

2 ) or the $2^{\text {nd }}$, we assume $\xi(t)$ to be given as a proper function of parameter $t$, then we should obtain a solution of ODE of the 1-st kind for $\mathrm{z}(t)$.

For example, if we choose the 2-nd way of above, we should obtain from (3.4):

$$
\begin{aligned}
& (\dot{z})^{2}=\frac{2 q_{1}(1-\mu)}{\sqrt{z(t)^{2}+r_{1}^{2}(x, y)}}+\frac{2 q_{2} \mu}{\sqrt{z(t)^{2}+r_{2}^{2}(x, y)}}+f \\
& r_{1}^{2}(x, y)=(\xi(t) \cdot \cos t+\mu)^{2}+(\xi(t) \cdot \sin t)^{2} \\
& r_{2}^{2}(x, y)=(\xi(t) \cdot \cos t-1+\mu)^{2}+(\xi(t) \cdot \sin t)^{2} \\
& f=C-\dot{\xi}^{2}(t) .
\end{aligned}
$$

We should also note that the question 'Will the spiral (*) converge to a fixed point or diverge to infinity?' should be researched additionally, depending on initial data of the proper case. So, the stability of a spiral motion is an open problem in celestial mechanics.

\section{CONCLUSION}

We have obtained a new type of exact solutions for photogravitational restricted three-body problem [1-3] (the case of spiral motion).

According to the Bruns theorem [4], there is no other invariants except well-known 10 integrals for three-body problem (including integral of energy, momentum, etc.). But in the case of restricted three-body problem, there is no other invariants except only one, Jacobian-type integral of motion $[5,6]$.

A key point is that we obtain the appropriate specific case of spiral motion from the Jacobian-type integral for photogravitational restricted three-body problem (when orbit of small 3-rd body is assumed to be like a spiral). Besides, we should especially note that there is a proper restriction to the type of spiral orbital motion of small 3-rd body, which could be possible for choosing as the exact solution of equations for photogravitational restricted three-body problem.

Let us demonstrate the proper asymptotic simplifications of the considered solutions; Eq. (3.5) could be simplified if we consider a quasi-planar case of orbital motion:

$$
(\dot{z})^{2}=\frac{2 q_{1}(1-\mu)}{r_{1}(x, y) \cdot \sqrt{1+\frac{z(t)^{2}}{r_{1}^{2}(x, y)}}}+\frac{2 q_{2} \mu}{r_{2}(x, y) \cdot \sqrt{1+\frac{z(t)^{2}}{r_{2}^{2}(x, y)}}}
$$

$$
\begin{aligned}
& +f \quad\left\{\frac{z(t)}{r_{1}} \rightarrow 0, \frac{z(t)}{r_{2}} \rightarrow 0\right\} \Rightarrow \\
& (\dot{z})^{2} \cong \frac{2 q_{1}(1-\mu)}{r_{1}(x, y)} \cdot\left(1-\frac{z(t)^{2}}{2 r_{1}^{2}(x, y)}\right) \\
& +\frac{2 q_{2} \mu}{r_{2}(x, y)} \cdot\left(1-\frac{z(t)^{2}}{2 r_{2}^{2}(x, y)}\right)+C-\dot{\xi}^{2}(t),
\end{aligned}
$$

$$
\frac{d z}{\sqrt{-\left(\frac{q_{1}(1-\mu)}{r_{1}^{3}(x, y)}+\frac{q_{2} \mu}{r_{2}^{3}(x, y)}\right) \cdot z(t)^{2}+\left(\frac{2 q_{1}(1-\mu)}{r_{1}(x, y)}+\frac{2 q_{2} \mu}{r_{2}(x, y)}+C-\dot{\xi}^{2}(t)\right)}}=d t
$$

where the left side of Equation (4.1) could be transformed to the proper elliptical integral [16] in regard to $z$.

The case below should be excluded from the variety of possible solutions:

$$
\begin{aligned}
& -\left(\frac{q_{1}(1-\mu)}{r_{1}^{3}(x, y)}+\frac{q_{2} \mu}{r_{2}^{3}(x, y)}\right) \cdot z(t)^{2}+ \\
& \left(\frac{2 q_{1}(1-\mu)}{r_{1}(x, y)}+\frac{2 q_{2} \mu}{r_{2}(x, y)}+C-\dot{\xi}^{2}(t)\right)=0 .
\end{aligned}
$$

Indeed, in such a case we could obtain from Eq. (3.5) that component $\mathrm{z}$ is under the linear dependence on the timeparameter $t$ (but we assumed: $\left\{\left(\mathrm{z} / r_{1}\right),\left(\mathrm{z} / r_{2}\right\} \rightarrow 0\right)$.

Besides, the appropriate restrictions of meanings of variables should be valid for all meanings of parameter $t \geq 0$ in (4.1) as below:

$$
\begin{aligned}
& -\left(\frac{q_{1}(1-\mu)}{r_{1}^{3}(x, y)}+\frac{q_{2} \mu}{r_{2}^{3}(x, y)}\right) \cdot z(t)^{2} \\
& +\left(\frac{2 q_{1}(1-\mu)}{r_{1}(x, y)}+\frac{2 q_{2} \mu}{r_{2}(x, y)}+C-\dot{\xi}^{2}(t)\right)>0 .
\end{aligned}
$$

Under a quasi-planar assumption above: $\left\{(\mathrm{z} / \mathrm{r}),\left(\mathrm{z} / \mathrm{r}^{2}\right)\right\}$ $\rightarrow 0$, it means that the proper restrictions at choosing of the spiral factor $\xi(t)$ should be given as below:

$$
\begin{aligned}
& \dot{\xi}^{2}(t)<\frac{2 q_{1}(1-\mu)}{\sqrt{\xi^{2}(t)+2 \xi(t) \cdot \mu \cdot \cos t+\mu^{2}}} \\
& +\frac{2 q_{2} \mu}{\sqrt{\xi^{2}(t)+2 \xi(t) \cdot(\mu-1) \cdot \cos t+(\mu-1)^{2}}}+C .
\end{aligned}
$$

For example, if $\xi(t) \gg 1$ we should obtain for the asymptotical final motions $t \rightarrow \infty$ (constants are chosen as below):

$$
\begin{aligned}
& \xi(t) \cdot \dot{\xi}^{2}(t)<\left\{2 q_{1}(1-\mu)+2 q_{2} \mu\right\}+\xi(t) \cdot C, \\
& C=0 \Rightarrow \xi(t)<\left(\frac{9}{2}\left(q_{1}(1-\mu)+q_{2} \mu\right)\right)^{\frac{1}{3}} \cdot\left(t+t_{0}\right)^{\frac{2}{3}}, \\
& \left(\frac{9}{2}\left(q_{1}(1-\mu)+q_{2} \mu\right)\right)=1, t_{0}=0, \Rightarrow \xi(t)<t^{\frac{2}{3}} .
\end{aligned}
$$


It means that we should choose a polinomial function with extent of time-parameter $t$ less than $<2 / 3$ as the spiral factor for the modelling of a spiral motion in such a case.

\section{DISCUSSIONS}

We obtain the appropriate specific case of a spiral motion for photogravitational restricted three-body problem from the Jacobian-type integral of motion (when orbit of small 3-rd body is assumed to be like $a$ spiral).

The main result, which should be outlined, is that in a case of quasi-planar orbital motion (of the small 3-rd body) the asymptotic expression for component $z$ of motion is proved to be given by the proper elliptical integral. But the elliptical integral is known to be a generalization of the class of inverse periodic functions.

Thus, by the proper obtaining of re-inverse dependence of a solution from time-parameter we could present the expression of $z(t)$ as a set of periodic cycles. So, the meaning of component $z(t)$ is proved to be limited in the proper range of values.

\section{CONFLICT OF INTEREST}

The author confirms that this article content has no conflict of interest.

\section{ACKNOWLEDGEMENTS}

I am thankful to CNews Russia project (Science \& Technology Forum, Prof. L.Vladimirov-Paraligon, Dr. A.Kulikov) - for valuable discussions in preparing of this manuscript.

Especially I am thankful to Dr. Badam Singh Kushvah for his valuable suggestions in preliminary discussions of this manuscript.

\section{REFERENCES}

[1] Radzievskii VV. The restricted problem of three bodies taking account of light pressure. Akad Nauk USSR Astron J 1950; 27 : 250.

[2] Shankaran, Sharma JP, Ishwar B. Equilibrium points in the generalized photogravitational non-planar restricted three body problem Int J Eng Sci Technol 2011; 3(2): 63-7.

[3] Ershkov SV. Yarkovsky effect in modified photogravitational 3bodies problem. IJPAM 2012; 78(3), 395-402.

[4] Bruns H. Ueber die Integrale der Vielkoerper-Problems. Acta Math Bd 1887; 11: 25-96.

[5] Szebehely V. Theory of Orbits. The Restricted Problem of Three Bodies. Academic Press New-York and London: Yale University, New Haven, Connecticut 1967.

[6] Duboshin GN. Nebesnaja mehanika. Osnovnye zadachi i metody. Moscow: "Nauka" (ru) (handbook for Celestial Mechanics) 1968.

[7] Douskos CN, Markellos VV. Out-of-plane equilibrium points in the restricted three body problem with oblateness. A\&A 2006; 446: 357-60.

[8] Shankaran SJP, Ishwar B. Out-of-plane equilibrium points and stability in the generalised photogravitational restricted three body problem. Astrophys Space Sci 2011; 332(1): 115-9.

[9] Chernikov YA. The photogravitational restricted three-body problem. Soviet Astron 1970; 14: 176.

[10] Kushvah BS, Sharma JP, Ishwar B. Nonlinear stability in the generalised photogravitational restricted three body problem with Poynting-Robertson drag. Astrophys Space Sci 2007; 312: 279-93.

[11] Radzievskii VV. A mechanism for the disintegration of asteroids and meteorites. Doklady Akademii Nauk SSSR 1954; 97: 49-52.

[12] Rubincam DP. Radiative spin-up and spin-down of small asteroids. Icarus 2000; 148: 2-11.

[13] Ershkov SV. The Yarkovsky effect in generalized photogravitational 3-body problem. Planetary Space Science 2012; 73(1): 2213.

[14] Singh J, Leke O. Stability of the photogravitational restricted threebody problem with variable masses. Astrophys Space Sci 2010; 326(2): 305-14.

[15] Varvoglis H, Hadjidemetriou JD. Comment on the paper "On the triangular libration points in photogravitational restricted threebody problem with variable mass" by Zhang, M.J., et al. Astrophys Space Sci 2012; 339(2): 207-10.

[16] Lawden D. Elliptic Functions and Applications. Springer-Verlag 1989. See also: http://mathworld.wolfram.com/EllipticIntegral.html

(c) Sergey V. Ershkov; Licensee Bentham Open.

This is an open access article licensed under the terms of the Creative Commons Attribution Non-Commercial License (http://creativecommons.org/licenses/by-nc/3.0/) which permits unrestricted, non-commercial use, distribution and reproduction in any medium, provided the work is properly cited. 